\title{
Non-Archimedean analytic curves in Abelian varieties
}

\author{
William Cherry ${ }^{\star}$ \\ Department of Mathematics, The University of Michigan, Ann Arbor, MI 48109-1003, USA \\ (Email: wcherry@math.lsa.umich.edu)
}

Received: 8 September 1993 /In revised form 2 February 1994

Mathematics Subject Classification (1991): 14G20, 14K15, 32P05, 32H25, $32 \mathrm{H} 20$

One of the main subtleties of non-Archimedean analysis is that the natural topology that one puts on non-Archimedean analytic spaces is totally disconnected, meaning that there is a base for the topology consisting of sets which are both open and closed. This makes it difficult, for instance, to define a good notion of analytic function so that one has analytic continuation properties. Of course one can make a sensible definition for what one means by an analytic function, and one of the more successful approaches has been the notion of a "rigid analytic function". This approach was started by Tate in [Ta], and a systematic introduction to rigid analysis is given in [BGR]. However, rigid analysis is very different from classical analysis in the sense that essentially none of the topological techniques which are commonplace in classical analysis can be used in rigid analysis. Recently, Berkovich, in [Ber], has come up with a new notion of non-Archimedean analytic spaces which have nice topological properties. For instance, Berkovich's spaces are locally arc-connected, locally compact, Hausdorff spaces. Using Berkovich's theory, one is able to add such topological techniques as covering spaces and map liftings to the study of nonArchimedean analysis. In his book, Berkovich proves the following theorem with the aid of the topological techniques his new theory encourages.

Theorem [Ber, 4.5.1] Let $X$ be the non-Archimedean analytification of a smooth algebraic curve of genus $\geqq 1$ defined over a field $K$, complete with respect to a non-trivial, non-Archimedean valuation. Then, every analytic map from the affine line $\mathbf{A}^{1}$ into $X$ is constant.

In this paper, I show that practically the same proof given by Berkovich for the above theorem shows that the only non-Archimedean analytic maps into

\footnotetext{
* Partially supported by Yale University and an Alfred P. Sloan Foundation Doctoral Dissertation Fellowship
} 
Abelian varieties are the constant maps. I will explain in Sect. 4 why this leads me to believe that the following question can be answered in the affirmative.

Question. Let $X$ be a non-singular projective algebraic variety defined over a field complete with respect to a non-trivial, non-Archimedean valuation. Is it true that there exists a non-constant analytic map from $\mathbf{A}^{1}$ into $X$ if and only if $X$ contains a rational curve?

In the case that an Abelian variety $A$ has degenerate reduction, there do exist non-constant analytic maps from $\mathbf{A}^{1 \times}$ (the affine line with the origin removed) into $A$, and I can show a non-Archimedean analogue to what is classically known over the complex numbers as Bloch's conjecture for analytic curves in subvarieties of Abelian varieties, or more generally in varieties with ample irregularity. (Bloch's conjecture was mostly proved over the complex numbers by Bloch and Ochiai [O]; a lemma of Green completes the proof. See also: Kawamata [Ka], Noguchi:-Ochiai [N-O], and Green-Griffiths [G-G]).

Theorem (Bloch's conjecture) Let $X$ be a smooth projective variety with ample irregularity, which by definition means that $\operatorname{dim} H^{1}\left(X, \mathcal{O}_{X}\right)$ is larger than $\operatorname{dim} X$, defined over a field complete with respect to a non-trivial, nonArchimedean valuation. Then, every non-Archimedean analytic map $f: \mathbf{A}^{1 \times} \rightarrow$ $X$ is contained in a proper algebraic subvariety.

In this paper, I will assume that the reader is acquainted with the main ideas in [Ber], or at the very least, with the basic theory of rigid analysis as in [BGR]. For a more leisurely exposition of the results presented in this paper, I refer the reader to my thesis, [Ch].

\section{Preliminaries}

Throughout this paper, $K$ will denote a field complete with respect to a nontrivial, non-Archimedean valuation. The prototypical example is the $p$-adic field $\mathbf{Q}_{p}$, which is the completion of $\mathbf{Q}$ with respect to the $p$-adic valuation ||$_{p}$ normalized so that $|p|_{p}=p^{-1}$. Another important field is the field $\mathbf{C}_{p}$, which is the completion of the algebraic closure of $\mathbf{Q}_{p}$. It is a theorem that $\mathbf{C}_{p}$ is algebraically closed, and the field $\mathbf{C}_{p}$ is the $p$-adic analogue of the complex numbers.

By analytic space, I will always mean analytic space in the sense of Berkovich as described in [Ber]; by $K$-analytic space, I will mean an analytic space defined over $K$. Berkovich's idea of analytic spaces has since been generalized in a couple of different directions. Berkovich himself gives a generalization in [Ber2]. For a different approach, a little more in analogy with schemes, see [Sch]. Since I will mainly be concerned with algebraic varieties, Berkovich's original theory will be more than sufficient for my purposes. Recall that each point in an analytic space corresponds to a bounded multiplicative semi-norm on the space of functions on some affinoid neighborhood. I will use the notation ||$_{x}$ to denote the semi-norm corresponding to a point $x$ in 
an analytic space $X$. Given an algebraic variety defined over $K$, one can provide it with a $K$-analytic structure in a completely straightforward manner; see [Ber] for details. I will denote both the algebraic variety and its analytification by the same symbol, and I doubt that this will lead to any confusion. Unless otherwise specified, all analytic spaces and maps will be defined over a fixed field $K$, complete with respect to a non-trivial, non-Archimedean valuation. The symbol $\mathbf{A}^{1}$ denotes the affine line, and the symbol $\mathbf{A}^{1 \times}$ denotes the affine line with the origin removed. The symbol $\mathbf{P}^{n}$ denotes projective $n$-space, and $\mathbf{P}^{1}$ therefore denotes the projective line. The symbol $\mathbf{B}^{n}$ will be used to denote the closed unit $n$-ball, which is the affinoid space associated to the Tate algebra $K\left\langle z_{1}, \ldots, z_{n}\right\rangle$, which is the ring of formal power series in $n$-variables with coefficients in $K$ whose absolute values tend to zero. The symbol $\mathbf{B}^{1}$ or simply $\mathbf{B}$ will denote the one dimensional closed unit ball. The open unit $\boldsymbol{n}$-ball is then defined by

$$
\stackrel{\mathbf{B}}{n}^{n}=\left\{x \in \mathbf{B}^{n}:\left|z_{j}\right|_{x}<1 \text { for } j=1, \ldots, n\right\} .
$$

The one dimensional open unit ball is denoted by $\mathbf{B}^{1}$ or simply $\mathbf{B}$.

In this paper, I will always assume that any affinoid space or subdomain is strictly affinoid. I will also assume that all analytic spaces are reduced and separated.

Recall that to each point $x$ in an analytic space $X$, one associates a field $\mathscr{K}(x)$, which is a complete, non-Archimedean extension of $K$, the field of definition for $X$. The field $\mathscr{K}(x)$ is obtained by first taking the ring of analytic functions on an affinoid neighborhood of $x$ modulo the kernel of ||$_{x}$ and then completing the fraction field of this ring with respect to ||$_{x}$. For an analytic function $f$, the notation $f(x)$ denotes the image of $f$ in $\mathscr{K}(x)$ under the canonical map. I denote by $X(K)$ the set of points $x \in X$ such that $\mathscr{K}(x)=K$. In the case that $K$ is algebraically closed, this space corresponds to the set of points in the rigid analytic space associated to $X$. For example, $\mathbf{A}^{1}(K)=K$, and $\mathbf{A}^{1 \times}(K)=K^{\times}$.

Throughout this paper, the use of the ${ }^{\sim}$ character will be reserved to denote a residue class. For instance, $\tilde{K}$ denotes the residue class field of $K$, which is the field defined by the elements in $K$ of norm $\leqq 1$ modulo the elements in $K$ of norm $<1$. In the case that $a$ is an element of $K$ with norm $\leqq 1$, then $\tilde{a}$ denotes the image of $a$ in $\tilde{K}$. We will also see that it is sometimes possible to associate to an analytic space $X$, a reduction $\tilde{X}$, which is an algebraic variety defined over the residue class field $\tilde{K}$. In this case, there will be a reduction map $\pi: X \rightarrow \tilde{X}$, so given a point $x$ in $X$, the notation $\tilde{x}$ is used to denote the image of $x$ under the reduction map $\pi$. Affinoid spaces $X$ have canonical reductions, and in the case that $X$ is affinoid, the notation will always refer to the canonical reduction.

We will need the following proposition, which is an elementary consequence of the theory of Newton polygons. (See, for instance, Amice [Am], for an introduction to the theory of Newton polygons.)

Proposition 1.1 Let $K$ be an algebraically closed field, complete with respect to a non-trivial, non-Archimedean valuation, and assume everything below is 
defined over $K$. Let $f: \mathbf{A}^{1} \rightarrow \mathbf{P}^{1}$ (resp. $f: \mathbf{A}^{1 \times} \rightarrow \mathbf{P}^{1}$ ) be an analytic map (i.e. a meromorphic function). Assume there exist points $x_{1}$ and $x_{2}$ in $\mathbf{P}^{1}(K)$ such that the sets $f^{-1}\left(x_{1}\right)$ and $f^{-1}\left(x_{2}\right)$ each contain at most a finite number of points. Then, $f$ is an algebraic map (i.e. a rational function). Furthermore, if $f^{-1}\left(x_{1}\right)$ and $f^{-1}\left(x_{2}\right)$ are both empty (resp. if the image of $f$ omits three points in $\left.\mathbf{P}^{1}(K)\right)$, then $f$ must be constant.

Recall that by an admissible affinoid cover $\mathscr{U}$ of an analytic space $X$, one means a cover $\mathscr{U}$ consisting of affinoid subspaces $U$ such that if $V$ is any affinoid subspace of $X$, then $\left.\mathscr{U}\right|_{V}=\{U \cap V\}$ is a finite covering of $V$. Let $U$ be an affinoid space. Let $V$ be an affinoid subdomain of $U$. If the induced morphism $\tilde{V} \rightarrow \tilde{U}$ is an open immersion, then $V$ is called a formal affinoid subdomain of $U$. Now let $X$ be an analytic space. An admissible, affinoid covering $\mathscr{U}$ of $X$ is called formal if the intersection $U \cap V$ is a formal affinoid subdomain of $U$ for every $U, V \in \mathscr{U}$. Given a formal covering $\mathscr{U}$ of $X$, one gets an algebraic variety $\widetilde{X}_{\mathscr{O}}$ over $\tilde{K}$ and a reduction map

$$
\pi_{\mathscr{U}}: X \rightarrow \widetilde{X_{\mathscr{U}}} .
$$

If $\mathscr{U}$ and $\mathscr{V}$ are two formal affinoid coverings of $X$, then the reductions $\widetilde{X_{\mathscr{U}}}$ and $\widetilde{X_{\mathscr{V}}}$ are in general non-isomorphic. However, two formal coverings $\mathscr{U}$ and $\mathscr{V}$ are called equivalent if $U \cap V$ is a finite union of formal subdomains of both $U$ and $V$ for every $U \in \mathscr{U}$ and every $V \in \mathscr{V}$. Equivalent formal coverings give rise to isomorphic reductions.

By an analytic group $G$, one means a group object in the category of analytic spaces. This means that there exist three morphisms

(a) $\mu: G \times G \rightarrow G$ (multiplication)

(b) $i: G \rightarrow G$ (inverse)

(c) $e: G \rightarrow G$ (identity)

satisfying the obvious relations. Note that $G$ itself is not a group, but it follows easily that $G(K)$ is a group. The two most important examples of analytic groups are the additive group $\mathbf{G}_{\mathrm{a}}$, which as an analytic space is isomorphic to $\mathbf{A}^{1}$, and the multiplicative group $\mathbf{G}_{\mathrm{m}}$, which as an analytic space is isomorphic to $\mathbf{A}^{1 \times}$. Another important analytic group is the affinoid analytic group $\mathbf{G}_{\mathrm{m}, 1}$ associated to the Tate algebra $K\left\langle z, z^{-1}\right\rangle$. The canonical reduction of $\mathbf{G}_{\mathrm{m}, 1}$ is the multiplicative group over the residue class field $\tilde{K}$.

A formal analytic space $X$ is an analytic space together with a fixed equivalence class of formal coverings. Such a space is denoted by $(X, \mathscr{U})$, where $\mathscr{U}$ is a formal covering of $X$ representing its equivalence class. A morphism

$$
\phi:(X, \mathscr{U}) \rightarrow(Y, \mathscr{V})
$$

of formal analytic spaces is a morphism $\phi: X \rightarrow Y$ of analytic spaces such that there exists a formal covering $\mathscr{U}^{\prime}$ of $X$ equivalent to $\mathscr{U}$ and a formal covering $\mathscr{V}^{\prime}$ of $Y$ equivalent to $\mathscr{V}$ such that for every $U \in \mathscr{U}^{\prime}$, there exists a $V \in \mathscr{V}^{\prime}$ such that $\phi(U) \subset V$. Recall that such a morphism induces a morphism

$$
\tilde{\phi}: \widetilde{X_{\mathscr{U}}} \rightarrow \widetilde{Y_{\mathscr{V}}},
$$


and this is the whole point of considering the category of formal analytic spaces. One easily sees that products exist in the category of formal analytic spaces, so it makes sense to talk about formal analytic groups, which are group objects in the category of formal analytic spaces.

There is an obvious functor from the category of formal analytic spaces to the category of analytic spaces, and from the category of formal analytic groups to the category of analytic groups, namely the functor which simply forgets the formal cover. By a theorem of Bosch [Bos], this functor from the category of formal analytic groups to the category of analytic groups is fully faithful, so in particular an analytic group can have at most one formal analytic group structure. Of course it might be that some analytic groups cannot be made into formal analytic groups at all, and in fact, this is the case. Note that the problem is not in finding a formal covering for an analytic group $G$, but rather that not all analytic groups $G$ have formal coverings such that the multiplication map from the product of $G$ with itself will then be a morphism in the category of formal analytic spaces. This last property is equivalent to the existence of a formal covering $\mathscr{U}$ such that $\widetilde{G_{\mathscr{U}}}$ is a group variety.

The term affine analytic torus, or affine torus, refers to a product of multiplicative groups $\mathbf{G}_{\mathbf{m}}$. (Actually, this should be called a split torus, but in this paper I will only be concerned with split tori.) The term affinoid torus will mean a product of the affinoid group $\mathbf{G}_{\mathrm{m}, 1}$. The rank of an affine or affinoid torus is by definition the number of copies of $\mathbf{G}_{\mathrm{m}}$ or $\mathbf{G}_{\mathrm{m}, 1}$ in the product. Let $T$ be an affine torus, and let $\Gamma$ be a torsion free, discrete subgroup of $T(K)$. Then, $\Gamma$ acts discretely and freely on $T$, so the quotient space $X_{\Gamma}=T / \Gamma$ is an analytic space. If the rank of $\Gamma$ is equal to the rank of $T$, then $X_{\Gamma}$ is called a complete analytic torus, or a complete torus.

\section{Non-Archimedean uniformization of Abelian varieties}

Over the complex numbers, every Abelian variety can be realized as $\mathbf{C}^{n}$ modulo a lattice. Over non-Archimedean ground fields, this is not at all the case. In this section, I summarize the non-Archimedean uniformization theory of Abelian varieties. For the details, see the work of Bosch and Lütkebohmert [BL2] and Sect. 6.5 of [Ber].

A formal analytic group $(G, \mathscr{U})$ is said to have Abelian reduction if $\widetilde{G_{\mathscr{H}}}$ is an Abelian variety, and $(G, \mathscr{U})$ is said to have semi-Abelian reduction if $\widetilde{G_{\text {YI }}}$ is a semi-Abelian variety; a semi-Abelian variety is an extension of an Abelian variety by an affine torus. In view of Bosch's result, [Bos], mentioned in the previous section, an analytic group $G$ will be said to have Abelian or semi-Abelian reduction if $G$ can be given the structure of a formal analytic group with Abelian or semi-Abelian reduction.

Theorem 2.1 (Semi-Abelian reduction) Let $A$ be an Abelian variety defined over a field $K$, complete with respect to a non-trivial, non-Archimedean vaiuation. Then, there exists a finite separable extension $K^{\prime}$ of $K$ and a unique 
compact subgroup $N$ of $A^{t}:=A \otimes K^{\prime}$ such that $N$ is an analytic domain in $A^{\prime}$ and is a formal analytic group with semi-Abelian reduction. Furthermore, $N$ contains a unique closed analytic subgroup $T_{1}$ in $N$ such that $T_{1}$ is an affinoid torus fitting into an exact sequence

$$
1 \rightarrow T_{1} \rightarrow N \rightarrow B \rightarrow 1
$$

where $B$ is a formal analytic group with Abelian reduction, which is the analytification of an Abelian variety. Note that an affinoid torus is a formal analytic group, so the exact sequence above reduces to an exact sequence defining $\tilde{N}$ as a semi-Abelian variety.

Theorem 2.2 (Uniformization theorem) Let $A$ be an Abelian variety defined over a field $K$, complete with respect to a non-trivial, non-Archimedean valuation. Let $K^{\prime}, A^{\prime}, T_{1}, N$ and $B$ be as in Theorem 2.1. Let $T$ be an affine analytic torus with the same rank as $T_{1}$, and embed $T_{1}$ into $T$ by

$$
T_{1} \cong \mathbf{G}_{\mathrm{m}, 1} \times \ldots \times \mathbf{G}_{\mathrm{m}, 1} \hookrightarrow \mathbf{G}_{\mathrm{m}} \times \ldots \times \mathbf{G}_{\mathrm{m}} \cong T .
$$

Then over $K^{\prime}$,

(a) $G=T \times N /$ diagonal exists as an analytic quotient, and there is an exact sequence

$$
1 \rightarrow T \rightarrow G \rightarrow B \rightarrow 1,
$$

so $G$ is a semi-Abelian variety. Here "diagonal" refers to the image of $T_{1}$ along the diagonal in $T \times N$.

(b) The immersion $N \hookrightarrow A^{\prime}$ extends uniquely to a surjective analytic group homomorphism

$$
\phi: G \rightarrow A^{\prime},
$$

which is also a topological covering map.

(c) $\Gamma=\operatorname{ker} \phi$ is a discrete subgroup in $G\left(K^{I}\right)$, which is free and whose rank is equal to the rank of $T_{1}$.

(d) $G$ is simply connected and $\pi_{1}\left(A^{\prime}\right) \cong \Gamma$.

\section{Analytic maps from $A^{1}$ and $A^{1 \times}$ into Abelian varieties and Bloch's conjecture}

This section begins by studying analytic maps from the affine line or the affine line minus a point into Abelian varieties. These results are then used to prove a non-Archimedean analogue of Bloch's conjecture for $\mathbf{A}^{1 \times}$. First, however, we need the following general lemma.

Lemma 3.1 Let $X$ be a formal analytic space defined over an algebraically closed field complete with respect to a non-trivial, non-Archimedean valuation. Assume that $\tilde{X}$, the reduction of $X$, does not contain any rational curves. Let $Y$ be a connected analytic subdomain of $\mathbf{P}^{1}$, and let $f: Y \rightarrow X$ be an analytic map. Then, the image of $f$ lies above a single closed point of $\tilde{X}$. 
Proof. First we show that the image of $f$ lies entirely above the closed points in $\tilde{X}$. Indeed, suppose there exists a point $y \in Y$ such that the point $x=f(y) \in X$ is such that $\tilde{x}$ is not a closed point of $\tilde{X}$. Then, there would be a non-zero homomorphism from $\mathscr{K}(x)$ into $\mathscr{K}(y)$. This would then imply

$$
\mathscr{K}(\tilde{x}) \hookrightarrow \widetilde{\mathscr{K}(x)} \hookrightarrow \widetilde{\mathscr{K}(y)},
$$

where the first inclusion follows directly from the definitions. However by Sect. 1.4.4 of [Ber], $\widetilde{\mathscr{K}(y)}$ is either $\tilde{K}$ or the field of rational functions in one variable over $\tilde{K}$, but since $\tilde{x}$ is assumed not to be closed, $\widetilde{\mathscr{K}(y)}$ must be the rational function field. This then gives a non-constant map from $\mathbf{P}_{\tilde{K}}^{1} \rightarrow \tilde{X}$, contradicting the assumption that $\tilde{X}$ contains no rational curves. Now since $Y$ is connected, $f(Y)$ is also connected. The anti-continuity of reduction (Corollary 2.4.2 in [Ber]) then implies that the image of $f$ cannot lie above more than one closed point in $\tilde{X}$.

The above lemma allows us to conclude that analytic maps from $\mathbf{A}^{1}$ or $\mathbf{A}^{1 \times}$ into Abelian varieties with Abelian reduction must be constant.

Theorem 3.2 Let $B$ be an Abelian variety defined over a field $K$, complete with respect to a non-trivial, non-Archimedean valuation. Assume that over a finite separable extension $K^{\prime}$ of $K, B^{\prime}:=B \otimes K^{\prime}$ can be provided with a formal analytic group structure and that as a formal analytic group, $B^{\prime}$ has Abelian reduction. If $f$ is an analytic map from $\mathbf{A}^{1}$ or $\mathbf{A}^{1 \times}$ into $B$, then $f$ must be constant.

Proof. It suffices to assume that $K$ is algebraically closed. Let $\pi: B \rightarrow \tilde{B}$ denote the reduction of $B$, so $\tilde{B}$ is an Abelian variety and therefore does not contain any rational curves. Since $\mathbf{A}^{1}$ and $\mathbf{A}^{1 \times}$ are connected, Lemma 3.1 implies that image of $f$ lies above a single closed point $\tilde{x}$ of $\tilde{B}$. Proposition 2.2 of [BL1] states that the inverse image under the reduction map of smooth closed points is analytically isomorphic to the open unit $n$-ball, and $\tilde{x}$ is smooth since $\tilde{B}$ is an Abelian variety. Thus,

$$
\pi^{-1}(\tilde{x}) \cong \stackrel{\mathbf{B}}{n}^{n},
$$

and $f$ can be considered as a map from $\mathbf{A}^{1}$ or $\mathbf{A}^{1 \times}$ into $\mathbf{B}^{n}$. Proposition 1.1 then implies that $f$ is constant since $z_{j} \circ f$ is bounded and hence constant for each coordinate function $z_{j}$ on $\stackrel{\mathbf{B}}{n}^{n}$.

Before looking at analytic maps into arbitrary Abelian varieties, I need to make a couple of observations about analytic maps from $\mathbf{A}^{1}$ and $\mathbf{A}^{1 \times}$ into affine tori.

Proposition 3.3 Every analytic map from $\mathbf{A}^{1}$ into an affine torus is constant.

Proof. Proposition 1.1 implies that any analytic map from $\mathbf{A}^{1}$ into $\mathbf{G}_{\mathrm{m}} \cong \mathbf{A}^{1 \times}$ is constant. Therefore, since affine tori are products of $\mathbf{G}_{\mathrm{m}}$, analytic maps from $\mathbf{A}^{1}$ into affine tori must therefore also be constant. 
Proposition 3.4 Let $T$ be an affine analytic torus, and let $f: \mathrm{A}^{1 \times} \rightarrow T$ be an analytic map. Then, $f$ is an analytic group homomorphism $\phi: \mathbf{G}_{\mathrm{m}} \rightarrow T$ composed with a translation $\tau: T \rightarrow T$.

Proof. Since $T \cong \mathbf{G}_{\mathrm{m}} \times \ldots \times \mathbf{G}_{\mathrm{m}}$, the map $f$ can be represented by an analytic map

$$
f: \mathbf{A}^{1 \times} \rightarrow \mathbf{G}_{\mathrm{m}} \times \ldots \times \mathbf{G}_{\mathrm{m}},
$$

given by

$$
z \mapsto\left(f_{1}(z), \ldots, f_{n}(z)\right),
$$

where

$$
f_{j}: \mathbf{A}^{1 \times} \rightarrow \mathbf{G}_{\mathrm{m}} \hookrightarrow \mathbf{A}^{1} .
$$

However, Proposition 1.1 implies that each function $f_{j}$ is in fact algebraic, and therefore the map is given by

$$
z \mapsto\left(c_{1} z^{d_{1}}, \ldots, c_{n} z^{d_{n}}\right),
$$

with $c_{j} \in K$ and $d_{j} \in \mathbf{Z}$. Therefore, $f$ is the composition of the group homomorphism

$$
z \mapsto\left(z^{d_{1}}, \ldots, z^{d_{n}}\right)
$$

followed by the translation

$$
\left(z_{1}, \ldots, z_{n}\right) \mapsto\left(c_{1} z_{1}, \ldots, c_{n} z_{n}\right) .
$$

Remark. This is markedly different from the complex analytic case, where the exponential function provides numerous maps from $\mathbf{C}^{\times}$into affine tori which are not translations of group homomorphisms.

Now we are ready to examine analytic maps from $\mathbf{A}^{1}$ and $\mathbf{A}^{1 \times}$ into arbitrary Abelian varieties. The proofs of the next two theorems are similar, so I state both theorems first and then give a joint proof.

Theorem 3.5 If $f: \mathbf{A}^{1} \rightarrow A$ is an analytic map into an Abelian variety, then $f$ must be constant.

Theorem 3.6 Let $X \subset A$ be a subvariety of an Abelian variety defined over a field $K$, complete with respect to a non-trivial, non-Archimedean valuation. If $f: \mathbf{A}^{1 \times} \rightarrow X$ is an analytic map, then the image of $f$ is contained in the translate of an Abelian subvariety $E$ of $A$, contained in $X$ and defined over a finite extension of $K$. Furthermore, $E$ does not have Abelian reduction over any field containing $K$ if $f$ is non-constant.

Proof of Theorems 3.5 and 3.6 Again, it suffices to assume that $K$ is algebraically closed. By Theorem 2.2 , there exists an Abelian variety $B$ with 
Abelian reduction, an affine analytic torus $T$, and an analytic group $G$ such that the diagram

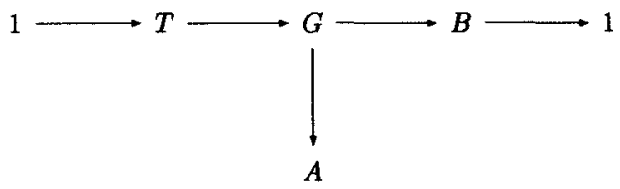

commutes, and the vertical arrow $G \rightarrow A$ is a universal covering map for $A$. This implies that the map $f: \mathbf{A}^{1}$ or $\mathbf{A}^{1 \times} \rightarrow A$ lifts to a map $h: \mathbf{A}^{1}$ or $\mathbf{A}^{1 \times} \rightarrow G$ as follows

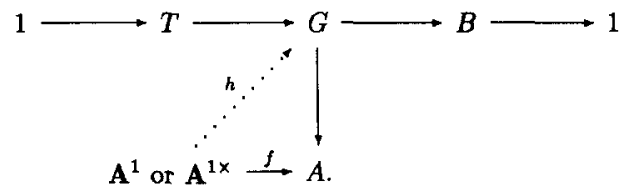

The image of either $\mathbf{A}^{1}$ or $\mathbf{A}^{1 \times}$ in $B$ is a point by Theorem 3.2. Therefore, the image of $h$ in $G$ is contained in a translate of $T$.

For Theorem 3.5 all that remains to say is that the map $h$ must then be constant by Proposition 3.3.

We now complete the proof of Theorem 3.6. Proposition 3.4 says that all analytic maps from $\mathbf{A}^{1 \times}$ into $T$ are translates of group homomorphisms, so the image of $h$ in $G$ is also the translate of a group homomorphism. Thus, the image of $f$ in $A$ is again the translate of a group homomorphism. Hence, the Zariski closure of the image of $f$ in $A$ is the translate of an Abelian subvariety $E$ in $A$ (see [L1, p. 84]). However, $X$ is closed in the Zariski topology, so this translate is contained in $X$.

Finally, Theorem 3.2 implies that $E$ does not have Abelian reduction if $f$ is not constant.

I conclude this section with the following non-Archimedean analogue of Bloch's conjecture.

Theorem 3.7 (Bloch's conjecture) Let $X$ be a smooth projective variety with ample irregularity, which by definition means that $\operatorname{dim} H^{1}\left(X, \mathcal{O}_{X}\right)$ is larger than $\operatorname{dim} X$. Then, every analytic map $f: \mathbf{A}^{1 \times} \rightarrow X$ is contained in a proper algebraic subvariety.

Proof. We may assume that the ground field $K$ is algebraically closed. Let $A$ be the Albanese variety of $X$ and $Y$ the image of $X$ in $A$. Then, by assumption, $Y$ is a proper subvariety of $A$, and $Y$ cannot be the translate of a proper Abelian subvariety since $Y(K)$ must generate $A(K)$ as a group. However, the image of $\mathbf{A}^{1 \times}$ in $Y$ is the translate of a proper Abelian subvariety of $A$ contained in $Y$ 
by Theorem 3.6. Therefore, the image of $f$ is contained in a proper subvariety of $Y$, and hence the image of $\mathbf{A}^{1 \times}$ under $f$ in $X$ is also contained in a proper subvariety since the map from $X$ to $A$ is algebraic.

Remark. Because the above theorem is only interesting in the case that the Albanese variety has degenerate reduction, I think it is somewhat artificial to look at analytic maps from $\mathbf{A}^{1 \times}$ into complete varieties. However, there are a number of theorems about holomorphic maps from $\mathbf{C}$ into open varieties which have good non-Archimedean analogies, at least provided that the nonArchimedean field $K$ has characteristic zero. For instance, one can show that every analytic map from $\mathbf{A}^{1 \times}$ into $\mathbf{P}^{n}$ which misses $2 n+1$ hyperplanes in general position must be constant, but that there exist non-constant analytic maps from $\mathbf{A}^{1 \times}$ into $\mathbf{P}^{n}$ missing $2 n$ hyperplanes in general position. This result (in either the complex case or the characteristic zero, non-Archimedean case) depends only on the Borel unit theorem, which is a triviality on $A^{1 \times}$ in the non-Archimedean case, and on projective linear algebra. Most other results of this type depend only on these two things, plus possibly the lemma on the logarithmic derivative from Nevanlinna theory, for which there is also a non-Archimedean analogue for $\mathbf{A}^{1 \times}$. See Chap. III of [Ch] for a collection of examples of results of this type.

\section{Lang's conjecture and open questions}

Recall that a complex analytic space $X$ is called Brody hyperbolic if the only analytic maps $f: \mathbf{C} \rightarrow X$ are the constant maps. Serge Lang has conjectured that this property is related to the Diophantine property of a variety being Mordellic and to the algebro-geometric property of a variety being of general type. Let $F_{0}$ be a field which is finitely generated over the rational numbers $\mathrm{Q}$, and let $X$ be a projective algebraic variety defined over $F_{0}$. Then, $X$ is said to be of general type, or pseudo canonical, if the canonical divisor class on $X$ is pseudo ample, which means that some multiple of it gives rise to a projective embedding of a Zariski open subset of $X$. Lang calls $X$ Mordellic if $X$ has only finitely many rational points over every field $F$ which is finitely generated over $F_{0}$. Then, one can state Lang's conjecture as follows: (see [L2, Conjecture VIII.1.2])

Conjecture 4.1 (Lang) The following conditions are equivalent for a projective variety $X$, defined over a subfield of the complex numbers which is finitely generated over the rationals.

(a) Considered as a complex analytic space, $X$ is Brody hyperbolic.

(b) $X$ is Mordellic.

(c) Every subvariety of $X$ is of general type (pseudo canonical). 
The principle behind this conjecture is that if a variety contains a subvariety which is the rational image of a group variety, then the variety will contain the image of a non-constant holomorphic map because the (Lie group) exponential maps give non-constant holomorphic maps into group varieties. Similarly, group varieties have infinitely many rational points once they have a rational point of infinite order because the group operation is algebraic. Therefore, the above conjecture in principle says that if any of the above three properties are true, then the variety contains no rational images of group varieties, and this should then imply the other two properties. Lang's conjecture is true for smooth projective curves: curves of genus $\geqq 2$ are Brody hyperbolic, which is an easy consequence of the uniformization theorem and Liouville's theorem; such curves are Mordellic by Faltings's theorem (the Mordell conjecture); and curves of genus $\geqq 2$ are clearly of general type since their canonical class is ample. Lang's conjecture is also true for subvarieties of Abelian varieties by a more recent theorem of Faltings, by Kawamata's structure theorem, and by Bloch's conjecture in the complex case.

I started looking at the questions addressed in this paper in order to better understand Lang's conjecture. However, in the non-Archimedean case, Abelian varieties behave as if they were hyperbolic. Therefore, studying the non-existence of non-constant analytic maps from $\mathbf{A}^{1}$ into non-Archimedean analytic spaces has not yet led to a greater understanding of Lang's conjecture. Nonetheless, it seems that there is a close relationship between the existence of non-constant non-Archimedean analytic maps and rational curves, and this is interesting in its own right. Whereas Lang conjectures that holomorphic curves distinguish between varieties with positive and non-positive canonical classes in the sense that the Zariski closure of the image of a non-constant holomorphic map from $\mathbf{C}$ should be a subvariety with non-positive canonical class, it appears that $p$-adic analytic curves distinguish between varieties with negative and non-negative canonical class, in the sense that it seems the Zariski closure of the image of an analytic map from $\mathbf{C}_{p}$ should be a variety with negative canonical class.

Lang's conjecture, together with the results above about analytic maps into Abelian varieties, inspires me to make the following conjecture:

Conjecture 4.2 Let $X$ be a non-singular projective algebraic variety defined over $\mathbf{C}_{p}$. Then, there exists a non-constant analytic map from $\mathbf{A}^{1}$ into $X$ if and only if $X$ contains a rational curve.

Theorem 3.5 together with the classification of algebraic surfaces implies that Conjecture 4.2 is true for algebraic surfaces $S$ such that either the Kodaira dimension of $S$ is $\leqq 1$ or the irregularity of $S$ is $\geqq 1$, leaving the case of general type surfaces with irregularity 0 open. See [Ch] for full details.

This paper is part of the author's Ph.D. thesis written under the supervision of Professor Serge Lang (Yale University). 


\section{References}

[Am] $Y$. Amice, Les nombres $p$-adiques. Presses Universitaires de France, 1975

[Ber] V. Berkovich, Spectral Theory and Analytic Geometry over Non-Archimedean Fields. AMS Surveys and Monographs 33, 1990

[Ber2] V. Berkovich, Étale Cohomology for Non-Archimedean Analytic Spaces. Draft 1993

[BGR] S. Bosch, U. Güntzer, R. Remmert, Non-Archimedean Analysis. Springer-Verlag, 1984

[BL,1] S. Bosch, W. Lütkebohmert, Stable Reduction and Uniformization of Abelian Varieties I. Math. Ann. 270 (1985), 349-379

[BL2] S. Bosch, W. Lütkebohmert, Stable Reduction and Uniformization of Abelian Varieties II. Invent. Math. 78 (1984), 257-297

[Bos] S. Bosch, Rigid Analytische Gruppen mit Guter Reduction. Math. Ann. 223 (1976), 193-205

[Ch] W. Cherry, Hyperbolic $p$-Adic Analytic Spaces. Ph.D. Thesis, Yale University, 1993

[F-P] J. Fresnel, M. van der Put, Géométrie Analytique Rigide et Applications. Birkhäuser, 1981

[G-G] M. Green, P. Griffiths, Two Applications of Algebraic Geometry to Entire Holomorphic Mappings. The Chern Symposium 1979, Proc. International Sympos, Berkeley, CA, 1979, Springer-Verlag, 1980, pp. 4l-74

[G-P] L. Gerritzen, M. van der Put, Schottky Groups and Mumford Curves. Lect. Notes Math. 817, Springer-Verlag, 1980

[Har] R. Hartshome, Algebraic Geometry. Springer-Verlag, 1977

[Ka] Y. Kawamata, On Bloch's Conjecture. Invent. Math. 57 (1980), 97-100

[L1] S. Lang, Introduction to Complex. Hyperbolic Spaces. Springer-Verlag, 1987

[L2] S. Lang, Number Theory III. Encycl. Math. Sci. 60, Springer-Verlag, 1991

[No] J. Noguchi, T. Ochiai, Geometric Funetion Theory in Several Complex Variables. Transl. Math. Monogr. 80, Am. Math. Soc., 1990

[o] T. Ochiai, On Holomorphic Curves in Algebraic Varieties with Ample Irregularity. Invent. Math. 43 (1977), 83-96

[Sch] P. Schneider, Points of Rigid Analytic Varieties. J. Reine Angew. Math. 434 (1993), $127-157$

[Ta] J. Tate, Rigid Analytic Spaces. Invent. Math. 12 (1971), 257-289 